\title{
A CROSS-DISCIPLINARY COMPARISON OF PUBLISHED ARTICLES: ADAPTED PHYSICAL ACTIVITY AND SPECIAL EDUCATION
}

\author{
Jihyun Lee \\ Sam Houston State University \\ Justin A. Haegele \\ Old Dominion University
}

\begin{abstract}
*Corresponding author: Jihyun Lee, Ph.D., Assistant Professor, Department of Kinesiology, Sam
Houston State University, email: jlee@ shsu.edu
\end{abstract}

\begin{abstract}
Documentary analyses of adapted physical activity (APA) literature have been conducted which discuss publication trends in the APA field (e.g., Karkeletsi, Skordilis, Evaggelinou, Grammatopoulou, \& Spanaki, 2012; Porretta \& Sherrill, 2005). However, few documentary analyses have compared those results with trends in other related fields, such as special education (SE). This study compares publication trends between APA and SE over a 10-year span. Primary journals from APA and SE, Adapted Physical Activity Quarterly (APAQ) and Exceptional Children (EC), respectively, were selected for a comparative documentary analysis. A total of 455 articles ( $A P A Q, \mathrm{n}=205 ; E C, \mathrm{n}=250$ ) from 2004 to 2013 were identified and coded based on the following categories: (a)country of the first author affiliation; (b)theoretical framework of empirical studies; (c)intervention research; (d)publication/research methods; (e) population of interest; and (f)topical areas. Results demonstrate similarities and differences between APA and SE publication trends. The results indicate that little intervention-based research was published across journals (14\% for $A P A Q ; 38 \%$ for $E C$ ). Both journals largely published group design research, and more single subject studies were observed in $E C$ $(\mathrm{n}=17)$ than in $A P A Q(\mathrm{n}=4)$. Additional similarities and differences in publication trends over the past ten years between two journals are discussed.
\end{abstract}

Keywords: adapted physical education, disabilities, theoretical framework, research methodology, disability studies

\section{INTRODUCTION}

Adapted physical activity (APA) can be defined as "research, theory, and practice directed toward persons of all ages underserved by the general sport sciences, disadvantaged in resources, or lacking power to access equal physical activity opportunities and rights" (Sherrill \& Hutzler, 2008, p.91). Reid (2003) expanded the definition of APA by referring to it as a cross disciplinary body of knowledge and practice used by professionals to serve and advocate not only for individuals with disabilities but also anyone experiencing movement difficulties. Thus, as a service delivery profession, APA influences various aspects of the lives of individuals with movement difficulties, including those involved in school-based physical education, rehabilitation, sports, leisure and other daily life contexts (DePauw \& Sherrill, 1994; Kudlacek \& Barrett, 2011; Reid \& Stanish, 2003).

Although historical records of APA practices are abundant and can be traced back to Ancient China (DePauw \& Sherrill, 1994), APA is still considered relatively new in terms of being a cross-disciplinary field of study (McCubbin, 2014). As an academic area, APA has seen a rapid expansion influenced by developments in both kinesiology and education (Hutzler, 2011; Sherrill, 2004). As such, many researchers have examined the development of APA by analyzing research 
and publication trends through documentary analyses of published materials (i.e., Lee \& Porretta, 2013; Karkaletsi et al., 2012; Porretta \& Sherrill, 2005; Reid \& Broadhead, 1995; Reid \& Prupas, 1998; Reid \& Stanish, 2003; Zhang, deLisle, \& Chen, 2006). These analyses discuss the current trends in research and predict future directions in APA.

Reid and Broadhead (1995) analyzed articles published in the first decade, Porretta and Sherrill (2005) analyzed articles published in the second decade, and Haegele, Lee, and Porretta (2015) analyzed research published in the third decade of Adapted Physical Activity Quarterly (APAQ) to describe characteristics of research and predict future directions. Reid and Prupas (1998) and Lee and Porretta (2013) analyzed 10 years of sport literature pertaining to individuals with disabilities to determine publication trends across seven research priority areas for disability sport that were developed by the Committee on Sports for the Disabled, of the U.S. Olympic Committee (DePauw, 1986). Zhang et al. (2006) reviewed abstracts from the special populations section of the American Alliance of Health, Physical Education, Recreation, and Dance (now known as the Society of Health and Physical Educators) national convention over a 36 year period to describe the overall picture of APArelated research during annual meetings. Reid and Stanish (2003) surveyed articles from APAQ and Clinical Kinesiology to determine whether APA can be considered an independent discipline or field of study. Most recently, Karkaletsi et al. (2012) identified research trends in APA by analyzing five years of articles published in $A P A Q$ (from 2006 to 2010).

Despite commonalities with other fields of study (e.g., kinesiology, physical education, and special education) and the efforts of researchers to analyze research and publication trends in APA, few analyses make comparisons between APA and related fields. Conducting comparative documentary analyses can discover important similarities or differences between APA and closely related fields. For example, Hutzler (2006) analyzed publications in $A P A Q$ and Physical Therapy and described the strengths and weaknesses of intervention research between the two journals. Hodge, Kozub, Robinson, and Hersman (2007) analyzed trends in identifying and describing participants in data-based studies. They compared data-based articles in $A P A Q$ with those in the Journal of Teaching in Physical Education to determine if shared trends exist in the identification and description of participants. Each of these comparative analyses provides recommendations for future APA publications (Hodge et al., 2007; Hutzler, 2006). However, further efforts to compare publication trends across fields are limited.

Researchers in APA are faced with several unique challenges which distinguish APA from other fields of study in kinesiology. For example, participants in APA research tend to be heterogeneous (Bouffard, 1993), and the research foci are greatly influenced by changing social demands including legislative change (Lavay \& Lasko-McCarthey, 1992). These challenges do not tend to influence publications or research for other kinesiology areas, such as physical education. However, researchers in disability-related disciplinary areas such as special education (SE) may share those challenges. As such, cross-disciplinary comparisons of publication trends between APA and SE may provide worthwhile information that can influence future APA scholarship. Therefore, the purpose of this documentary analysis was to examine and compare trends in APA and SE publications over a ten-year span.

For the purposes of this analysis, the academic journals $A P A Q$ and Exceptional Children (EC) were selected to represent publications in APA and SE, respectively. The journals were selected based on several criteria. Both $A P A Q$ and $S E$ are considered international, scholarly journals in their respective field. Both are the official journals of highly regarded international organizations; $E C$ is the official journal of the Council for Exceptional Children and $A P A Q$ is the official journal of the International Federation for Adapted Physical Activity. Each journal publishes research, research reviews, and databased position papers and does not publish practical application papers. $A P A Q$ and $E C$ 
both publish general disability-related content, rather than content specific to one disability (e.g., Journal of Visual Impairment \& Blindness). In addition, $A P A Q$ is considered the leading international journal in APA research (Porretta \& Sherrill, 2005) and has been found to contain articles by the highest quality and most prolific APA scholars (O'Connor, French, Sherrill, \& Babcock, 2001), while $E C$ is regarded as the highest ranked and most widely cited special education print journal (Mastropieri \& Scruggs, 2014).

\section{METHOD}

\section{Data Source}

A total of 455 publications $(A P A Q \mathrm{n}=205 ; E C$ $\mathrm{n}=250)$ from 2004 to 2013 were accessed electronically for this analysis. This timespan was selected to compare a recent snapshot of publications across the two disciplines and is consistent with timespans of previous documentary analyses (e.g., Porretta \& Sherrill, 2005). The investigators, experts in APA, screened all published articles in each issue of each journal. Articles were searched for peer-reviewed, full-length publications; editorials and books and media review articles were not considered. Procedures were followed based on Duncan's (1989) suggestions for document analyses, which include: (a)establishing coding categories and subcategories, (b)pre-testing the data using those categories, (c)revising the categories and subcategories based on the pre-test data if necessary, (d)re-testing if necessary, (e)coding data, and (f)calculating reliabilities. Similar procedures were used by Johnson and Kittleson (2000) and Zhang et al. (2006) in their analyses.

\section{Categories and Subcategories of Analysis}

Categories of analysis are descriptive phrases that are developed to analyze contents of documents (Zhang et al., 2006). For the purposes of this analysis, six categories were identified: (a)country of the first author affiliation; (b)theoretical framework in empirical studies; (c)intervention; (d)publication/research methods; (e)population of interest; and (f)topical areas. Each of the categories was developed as an area of interest for comparison between publications in APA and SE. Several categories (i.e., theoretical framework in empirical studies, intervention, research methods) were selected because of interest in whether common recommendations made in APA would reflect in current publication trends in APA or SE.

Each category contained several relative subcategories or components of a category that were used for the systematic coding process (Duncan, 1989). The number of subcategories demonstrated the breadth of a category so that no relevant information was left un-coded and each subcategory was mutually exclusive (Zhang et al., 2006). The subcategories and the coding process are presented in the next section.

\section{Systematic Coding Procedure}

The investigators used six categories with subcategories as criteria to code each article accessed. Each article was assigned only one subcategory within each designated category. Thus, each article was coded six times, once per category.

Two investigators served as coders and also recoded the data for interrater and intrarater reliabilities. The first investigator coded all $A P A Q$ articles. The second investigator coded $25 \%$ of randomly selected $A P A Q$ articles. In addition, the first investigator re-coded $25 \%$ of randomly selected $A P A Q$ articles. The second investigator coded all $E C$ articles. The second investigator recoded $25 \%$ of randomly selected $E C$ articles. The first author then re-coded $25 \%$ of randomly selected EC articles. The coded and re-coded data by the two investigators across both journals were analyzed to determine an estimate of interrater and intrarater agreement.

The first category of analysis was the first author-country affiliation. This category of analysis included seven categories, United States, Canada, European countries, Asian countries, South American countries, 
Australia/ Oceania, and African Countries. The United States and Canada could be conceptualized as one category-North America-but the large number of articles from these countries warranted splitting the category for more specificity. The country of affiliation was determined using the university affiliation of the first author (Ward \& Ko, 2006). The first five subcategories for the author-country affiliation category were for articles published by first authors affiliated with universities in the United States, Canada, European Countries, Asian countries, and South American countries. The sixth was for articles published by first authors affiliated with universities in Australia and countries considered Oceania, including New Zealand. Lastly, the seventh subcategory was used for authors affiliated with countries located in Africa.

The second category of analysis, theoretical framework in empirical studies, included three subcategories, theory present, theory not present, and not applicable. The first subcategory was used when authors explicitly described and discussed a theoretical or conceptual framework within the text of an empirical research article. The second subcategory was used when there was an absence of a discussion of a theoretical or conceptual framework within the text of an empirical research article. The third subcategory, not applicable, was used when the article was not an empirical research article. For the purposes of this analysis, empirical research refers to studies that contain original or replicated data (Thomas, Nelson, \& Silverman, 2005). Therefore, documentary analyses, meta-analyses, literature reviews, and conceptual papers were not considered empirical. This strategy was used because of the interest in whether theoretical considerations were taken in empirical research only. The inclusion of nonempirical articles such as viewpoint articles within this categorization would inflate the number of articles that are theory-based and would, in turn, mislead the investigators about the results.

research, included two subcategories, intervention research and non-intervention research. The first subcategory was used when a study sought to determine a causal or functional relationship between independent and dependent variables by systematically manipulating independent variables for a predetermined period of time (Thomas et al., 2005). The second subcategory was used for all articles that did not meet the criteria for the first subcategory.

The fourth category, publication/research method, included nine subcategories: (a)group design, (b)single subject, (c)qualitative, (d)case study, (e)mixed methods, (f)document analysis, (g)literature review, (h)meta-analysis, and (i)conceptual. The first subcategory was used if the study aggregated or synthesized empirical data across participants and used various statistical procedures for data analysis (Gay, Mills, \& Airasian, 2006). The second subcategory was for studies where each participant served as his or her own control and experienced both the control and treatment conditions (Johnston \& Pennypacker, 2009). The third subcategory was for studies that collected, analyzed, and interpreted narrative and/or visual data using a qualitative research method to explore phenomena (Gay et al., 2006). The fourth subcategory centered on quantitative, descriptive research studies which gathered large amounts of information on a few participants (Thomas et al., 2005). This subcategory did not include qualitative case studies, which were entered into the second subcategory. Studies that included a combination of more than one empirical research method (typically both group designs and qualitative methodologies) made up the fifth subcategory. The sixth subcategory was used for articles that systematically analyzed written contents of communications, such as textbooks, essays, or published articles (Fraenkel, Wallen, \& Hyun, 2012). The seventh subcategory was for articles that included a systematic identification, location, and analysis of documents related to a specific research question (Gay et al., 2006). Papers that involved similar characteristics to literature reviews, but also contained a definitive methodology concerning decisions 
in the literature analysis and quantified results of reviewed articles in a standard metric such as effect size, were in the eighth subcategory (Thomas et al., 2005). The last subcategory, conceptual, included articles which did not fit into any of the previous categories and presented an opinion or viewpoint of the author(s).

The fifth category, population of interest, included ten subcategories: (a)autism spectrum disorder (ASD), (b)intellectual disabilities, (c)sensory impairment, (d)learning disabilities, (e)physical disabilities, (f)multiple disabilities, (g)other disabilities, (h)stakeholders, (i)non-disabled peers, and (j)field wide. The first subcategory included articles which focused on individuals diagnosed with ASD. The second subcategory was employed when articles included individuals with intellectual disabilities including Down syndrome. The third subcategory coded articles that included individuals with sensory impairments including those with hearing impairments, visual impairments, and who are blind. The fourth subcategory was used for articles which described participants having learning disabilities or being at-risk for delays. The fifth subcategory was used when articles included individuals with physical disabilities such as those with amputations, cerebral palsy, muscular dystrophy, spinal injuries, or other disabilities which cause physical limitations. The sixth subcategory was used for studies which focused on individuals with multiple disabilities. The seventh subcategory was for studies which included individuals with disabilities that did not fit within the previously described categories. The eighth subcategory included studies which focused on stakeholders, including parents of children with disabilities, teachers, and other school personnel. The ninth subcategory was used for studies which focused on school-aged individuals without disabilities. The last subcategory, field-wide, was used when studies dealt with content which was not applicable to a specific population.

The sixth category, topical focus, included seven subcategories based on Lee and Porretta (2013): (a)exercise sciences; (b)psychology; (c)educational approaches; (d)technology; (e)stakeholder training; (f)instrument validation; and (g)research trends. Thus operational definitions of each subcategory were in accord with Lee and Porretta (2013). The first subcategory was used for articles which focused on physiology, biomechanical, medical, health, or physical activity-related topics. Articles were entered into the second subcategory that focused on psychological, sociological, philosophical, legislative, or attitudinal aspects of adapted physical activity. The third subcategory was used for articles that discussed educational approaches and policy for individuals with disabilities. The fourth subcategory was employed when articles focused on technological advances. The fifth subcategory was used for articles pertaining to training of stakeholders such as parents, staff, teachers or peers. The sixth subcategory was reserved for articles discussing instrument validations including establishing reliabilities. Lastly, the seventh subcategory was used when articles discussed research trends or methodologies.

\section{Data Analyses}

Data were analyzed using frequencies and percentages. A frequency was the summed number of articles coded in a given subcategory in each journal from 2004-2013. A percentage was computed by the summed number of articles coded in a given subcategory divided by the articles coded and then multiplied by 100 . The percentage indicates the proportion of a subcategory in its category across the breadth of research. Research trends were based on yearly frequencies or the summed number of articles coded in a specified subcategory in a given year (Zhang et al., 2006). Yearly frequencies were displayed using line graphs and data were analyzed using visual analysis. Researchers compared percentages, frequencies, and trends across $E C$ and $A P A Q$.

\section{RESULTS AND DISCUSSION}

The calculated interrater and intrarater reliabilities for $A P A Q$ yielded $91 \%$ and $96 \%$ 
agreement, respectively. The calculated interrater and intrarater reliabilities for $E C$ yielded $96 \%$ and $96 \%$ agreement, respectively. These coefficients indicate that the number of articles identified and coded reached acceptable estimates of reliability (Cooper, Heron, \& Heward, 2007). Therefore, it was considered that the investigators applied the categories and subcategories consistently.

The following sections describe and discuss results of this analysis. Results provide a comparison of publication trends between $A P A Q$ and $E C$ over a ten-year period with several notable features. Several similarities and differences of interest between the two academic journals are highlighted and discussed.

\section{Country of the First Author Affiliation}

Of the publications analyzed, the highest percentage of articles across both publications is those published by first authors associated with universities in the United States. First authors from the United States accounted for $43 \%$ and $93 \%$ of $A P A Q$ and $E C$ publications, respectively. For $A P A Q$, first authors affiliated with Canadian (24\%) and European (20\%) universities also contributed a large percentage of publications over the ten-year period. Authors affiliated with Asian (5\%), Australian/Oceania (4\%), South American (2\%), and African ( $<1 \%)$ universities published less over that time frame. Conversely, countries outside of the United States were far less represented in EC. Authors associated with European (4\%), Australian/Oceanic (2\%), Canadian (1\%), and Asian (1\%) combined for only $7 \%$ of $E C$ articles over the ten-year span. There were zero articles in $E C$ found by authors from South American of African countries.

While first authors affiliated with universities in the United States contributed the highest percentage of publications across both journals, there were differences between journals across the ten-year span in terms of authorship. Specifically, in addition to the large percentage of authors from the United States, those from universities in Canada and Europe also contributed notable percentages of
$A P A Q$ publications $(24 \%$ and $20 \%$, respectively). In contrast, only $1 \%$ of $E C$ articles came from authors from Canadian institutions and 4\% from European institutions. Further, whereas approximately $12 \%$ (combined) of $A P A Q$ articles were contributed to by authors in Asian, South American, Australian/Oceanic, and African countries, this group only contributed $3 \%$ of $E C$ papers. Therefore, the results of this analysis suggest that publications in $A P A Q$ demonstrate a wider range of contributors across different countries than publications in $E C$, where the majority of articles were written by first authors at institutions in the United States.

There are several potential explanations why trends in country of affiliation exist. First, scholars in APA have stressed the importance of an international representation of research in $A P A Q$ over the past few decades (Porretta \& Sherrill, 2005), which may contribute to the high percentage of contributions from authors outside of North America. Secondly, $A P A Q$ is one of few international journals focusing on APA (e.g., another being European Journal of Adapted Physical Activity). However, there are many academic journals that focus on SE, some of which are specific to geographic areas (e.g., European Journal of Special Needs Education, British Journal of Special Education) or international (e.g., International Journal of Early Childhood Special Education). The availability of additional journals, specifically those that are internationally-based, provides more options for those scholars who are based outside of North America for SE publications. Third, although the Council of Exceptional Children is considered an international organization, there are several other international organizations which focus on special education (e.g., International Association of Special Education) which also provide opportunities for scholarly activity for members. This, again, could pull authors from various countries in different directions in regard to publication destinations. On the other hand, the International Federation of Adapted Physical Activity, with promotes APAQ, is the only major global organization which is 
specific to the field of APA and attracts attention from scholars worldwide. Finally, the upward trend of publications from individuals representing universities in European countries may be attributed to the growth of APA as an academic discipline in Europe, including the continued influence of the European Federation of Adapted Physical Activity and the development of the European Journal of Adapted Physical Activity (Kudlacek \& Barrett, 2011).

\section{Theoretical Framework in Empirical Studies}

Of the $205 A P A Q$ and $250 E C$ publications screened, the majority of publications were identified as being empirical articles which did not explicitly address a theoretical or conceptual framework in the text. Of the 205 $A P A Q$ articles, 103 (50\%) were empirical articles which did not explicitly describe a theoretical framework, 72 (35\%) were considered empirical articles that did explicitly describe a theory, and 30 articles (15\%) were considered non-empirical articles. Of the 250 $E C$ publications, 132 of the 250 (53\%) were empirical articles that did not explicitly describe the use of a theory or conceptual framework, $39(16 \%)$ were empirical and included a theoretical framework, and 79 articles $(32 \%)$ were considered non-empirical. It should be noted that the majority of both $A P A Q$ and $E C$ publications were considered empirical. $A P A Q(85 \%)$ showed a higher percentage of empirical studies than $E C$ $(68 \%)$.

This analysis demonstrates that empirical articles in $A P A Q$ tend to employ and describe theoretical or conceptual frameworks at a higher rate than articles published in $E C$. One such explanation which may contribute to this difference is that scholars in APA have explained the importance of theoretical or conceptual frameworks throughout the short history of the field (Cervantes \& Taylor, 2011; Haegele \& Porretta, 2015; Porretta \& Sherrill, 2005). For example, Haegele and Porretta (2015) recently explained the importance of theoretical or conceptual frameworks as being related to the development of the research hypothesis and that models and frameworks can either be confirmed or revised for further use based on those empirical results.

Although publications in $A P A Q$ tend to use theoretical or conceptual frameworks more often than those in EC, $50 \%$ or more publications in both journals did not explicitly present a theoretical or conceptual framework. The percentage of publications without an explicitly described theory/concept is inflated when only empirical studies are considered (59\% APAQ and $77 \%$ EC). This is somewhat surprising because of the aforementioned arguments of APA researchers for the importance of theory-driven research (e.g., Cervantes \& Taylor, 2011; Haegele \& Porretta, 2015; Porretta \& Sherrill, 2005). In addition, according to Karkaletsi et al. (2012), results of a recent documentary analysis indicated that the use of theoretical/ conceptual frameworks in $A P A Q$ had been increasing in recent years. These researchers reported that over $57 \%$ of the $A P A Q$ articles over a recent five year period were grounded in a theory. This figure conflicts with the current analysis which found only $35 \%$ of $A P A Q$ articles to describe a theory in the text. This difference has several explanations. First, the current study attempted to determine the percentage of empirical research that explicitly described a theoretical framework, rather than publications in general. Second, consistent with investigations by previous researchers (Haegele \& Porretta, 2015; Porretta \& Sherrill, 2005), only the articles that explicitly stated a theory in the text were considered theoretically based. This rigorous procedure may have contributed to findings of the current analysis being lower than that of the previous analysis. Still, the results of the current analysis agree with findings from most recent research regarding APA publications that there may be a dearth in theory-based research (Haegele et al., 2015; Reid \& Stanish, 2003). Although APA scholars have declared the importance of theoretical/conceptual underpinnings in research, there are explanations why this type investigation may be absent. Reid and Stanish (2003) suggest that theoretical/conceptual frameworks may be omitted from empirical studies in APA 
because researchers tend to be biased toward applied research which aims to address immediate and specific issues which practitioners face. This explanation can hold equal weight for researchers publishing in $A P A Q$ as well as those in $S E$. In agreement with authors of previously published papers, the authors of this article encourage future researchers in APA to consider framing studies within theoretical or conceptual frameworks to enhance research in our field.

\section{Publication/Research Methods}

Table 1. Publication/ Research Methods across journals.

\begin{tabular}{ccccc}
\hline Subcategory & \multicolumn{2}{c}{$A P A Q$} & \multicolumn{3}{c}{$E C$} \\
\hline & Frequency & Percentage & Frequency & Percentage \\
\cline { 2 - 5 } Group & 127 & $62 \%$ & 129 & $52 \%$ \\
Qualitative & 34 & $17 \%$ & 22 & $9 \%$ \\
Conceptual & 16 & $8 \%$ & 34 & $13 \%$ \\
Literature Review & 7 & $3 \%$ & 21 & $8 \%$ \\
Mixed-Methods & 7 & $3 \%$ & 2 & $<1 \%$ \\
Document Analysis & 6 & $3 \%$ & 15 & $6 \%$ \\
Single Subject & 5 & $2 \%$ & 18 & $7 \%$ \\
Case Study & 3 & $1 \%$ & 0 & $0 \%$ \\
Meta-Analysis & 0 & $0 \%$ & 9 & $4 \%$ \\
\hline
\end{tabular}

Table 1 identifies the frequencies and percentages of publications across $A P A Q$ and $E C$ over the ten-year span by publication/research methods. For both journals, group design studies were most common with 127 articles (62\%) for $A P A Q$ and $129(52 \%)$ for $E C$. Other empiricalresearch methods accounted for lower percentages, with qualitative studies being the second most common (17\% for $A P A Q ; 9 \%$ for $E C)$. Further, both journals demonstrated low percentages $(<4 \%)$ of publications categorized as mixed-methods and case studies.

One discrepancy between journals is the number of articles which were categorized in non-empirical categories (i.e., document analysis, literature review, meta-analysis, and conceptual). Seventy-nine publications (32\%) in $E C$ were entered into one of these four categories with conceptual $(n=34)$ and literature review $(n=21)$ being the most common. In contrast, only 29 publications (14\%) from $A P A Q$ were considered one of the four non-empirical research categories, 16 of which (8\%) being considered conceptual. This difference may be related to the youth and developmental stage of APA as a field of study. In comparison to other fields of study in education, such as SE, APA is considered relatively new (McCubbins, 2014) and is still expanding as a field of knowledge (Lavay \& Lasko-McCarthey, 1992). Therefore, much research is being conducted in the field which may not have a previous body of literature. However, since SE may be more fully developed as a field of study, there may be richer bodies of literature which would warrant reviews of literature, documentary analyses, meta-analyses, and other papers which look at research historically.

Another difference was found in the number of articles which used a single subject research design. Only 5 publications $(2 \%)$ in $A P A Q$ were categorized as single subject designs, whereas 18 (7\%) studies in $E C$ fit this categorization. The higher number of single subject designs in SE research is not surprising, since single subject designs are known to fit many of the research questions used in SE and have been suggested as an appropriate research methodology for documenting evidence-based practices in SE (Horner et al., 2005). One of the common challenges between SE and APA research is the heterogeneity of participants (Bouffard, 1993). Byiers, Reichle, and Symons (2012) 
argued that single subject research designs are more suitable for demonstrating internal validity/experimental control than group designs when dealing with small and/or very heterogeneous populations. Further, APA scholars throughout the brief history of the field have recommended further use of these designs when engaging in APA research (e.g., Bouffard, 1993; Watkinson \& Wasson, 1984). The challenge of the heterogeneity of participants and suggestions from APA scholars may warrant future APA researchers to consider the single subject research paradigm when designing and implementing research.

\section{Intervention}

Despite the large number of empirical studies in both journals, only small percentages of publications used an intervention approach. Of the $205 A P A Q$ publications, only 25 (12\%) were considered interventions. For $E C, 38$ $(15 \%)$ publications were considered interventions. Therefore, most publications across both journals ( $88 \%$ for $A P A Q ; 85 \%$ for $E C$ ) were considered categorized as not being intervention research.
These results confirm previous conclusions that research in APA has been largely observational in nature (Zhang et al., 2006). It also suggests that SE research, such as that found in $E C$, may be observational in nature as well because of the small number of studies that were considered interventions. However, since there are numerous publication options for SE research, some of which focus specifically on intervention research (e.g., Journal of Positive Behavior Interventions; Intervention in School and Clinic), it is more difficult to make that conclusion for the SE field. Of note is that of the 38 intervention studies in EC, 18 (47\%) were considered single subject designs. The high percentage of single subject designs in intervention research in SE reinforces the value of using this research paradigm in studies pertaining to heterogeneous populations. Again, this may warrant further use of this research paradigm in APA related research.

\section{Population of Interest}

Table 2. Frequency and percentage of population of interest across journals.

\begin{tabular}{ccccc}
\hline Subcategory & \multicolumn{2}{c}{$A P A Q$} & \multicolumn{2}{c}{$E C$} \\
\hline & Frequency & Percentage & Frequency & Percentage \\
\cline { 2 - 5 } Physical Disabilities & 51 & $25 \%$ & 1 & $<1 \%$ \\
Intellectual Disabilities & 37 & $18 \%$ & 14 & $6 \%$ \\
Learning Disabilities & 25 & $12 \%$ & 48 & $19 \%$ \\
Stakeholders & 21 & $10 \%$ & 49 & $20 \%$ \\
Field Wide & 21 & $10 \%$ & 42 & $17 \%$ \\
Multiple Disabilities & 14 & $7 \%$ & 39 & $16 \%$ \\
Other Disabilities & 11 & $5 \%$ & 12 & $5 \%$ \\
ASD & 10 & $5 \%$ & 10 & $4 \%$ \\
Sensory Impairments & 9 & $4 \%$ & 4 & $2 \%$ \\
Non-Disabled Peers & 6 & $3 \%$ & 31 & $12 \%$ \\
\hline
\end{tabular}

Table 2 describes the frequencies and percentages of publications across $A P A Q$ and $E C$ over the ten-year span by population of interest. Differences between the two journals were more evident than similarities in regard to population of interest.

The investigators anticipated high percentages of ASD-related publications in both journals, based on the recently garnered international attention of ASD as a disability 
category of interest due to current spikes in diagnoses (Johnson, 2014). However, a low amount of articles pertaining to ASD were found across journals. Both $E C$ and $A P A Q$ which focused on this disability population warranted only 10 publications apiece. Several hypotheses might be generated in regard to the results. First, difficulties in determining homogeneous groups due to the largely individualized characteristics of ASD might have contributed to the dearth in ASD-related research. Second, accurate ASD diagnoses are known to be difficult to obtain, particularly when differentiating between ASD and other, similar disabilities such as Asperger's syndrome (Obrunsnikova \& Rocco Dillon, 2011). Another possible explanation is that scholars interested in research regarding ASD may choose other, ASD- focused publications for their research outlet such as the Journal of Autism and Developmental Disorders and Focus on Autism and Other Developmental Disabilities. Therefore, journals such as $A P A Q$ and $E C$, which publish materials across diverse disability categories, may not receive many manuscripts specific to this disability.

Several differences were found between $A P A Q$ and $E C$ with respect to population of interest as well. There was a large difference between journals in regard to the percentage of publications pertaining to individuals without disabilities, including stakeholders, non-disabled peers, and field wide articles. For $E C$, these three categories combined accounted for 122 of the 250 (49\%) of articles analyzed, whereas only 48 of 205 (23\%) of $A P A Q$ articles were focused on those without disabilities. The high number of articles pertaining to stakeholders (e.g., teachers, parents; $n=49$ ) and non-disabled peers $(n=31)$ may be related to the high volume of education-based publications in $E C$ $(n=150)$.
Second, there were large discrepancies in several disability categories between journals. The largest percentage for the target population in $A P A Q$ publications were categorized as physical disabilities $(25 \%)$, while this category was the smallest percentage in EC $(<1 \%)$. Another large percentage category in $A P A Q$, intellectual disabilities $(37 \%)$, was ranked sixth in $E C$ $(6 \%)$. Of the more common population of interest for $E C$ were individuals with learning disabilities (19\%) and multiple disabilities $(16 \%)$. These categories were shown as having lower percentages in $A P A Q$ (12\% and $7 \%$ respectively). Publications focusing on learning disabilities may be more prevalent in $E C$ because more educationally focused publications found in that journal.

It should be noted that findings in regard to population of interest in this analysis conflict with recent findings by Karkaletsi et al. (2012), which include overlapping time periods. These differences may be attributed to differences in subcategories as well as the five additional years of $A P A Q$ included in this analysis. For example, Karkaletsi and colleagues included only 11 articles into a generic category, which included all content that was not disability specific, whereas nondisability specific categories in this analysis, including those categorized stakeholders $(n=21)$, non-disabled peers $(n=6)$, and field wide $(n=21)$, accounted for 48 articles. Further, other articles that may have been categorized as disability specific (i.e., hearing impairment) by Karkaletsi et al. may have been categorized in the stakeholder subcategory in the current analysis if the authors determined the focus population was a parent or teacher rather than the student.

\section{Topical Focus}


Table 3. Frequency and percentage of topical focus across journals.

\begin{tabular}{ccccc}
\hline Subcategory & \multicolumn{2}{c}{$A P A Q$} & \multicolumn{3}{c}{$E C$} \\
\hline & Frequency & Percentage & Frequency & Percentage \\
\cline { 2 - 5 } Exercise Sciences & 66 & $32 \%$ & 3 & $1 \%$ \\
Psychology & 65 & $32 \%$ & 68 & $27 \%$ \\
Educational Approaches & 31 & $15 \%$ & 150 & $60 \%$ \\
Instrument Validation & 27 & $13 \%$ & 8 & $3 \%$ \\
Research Trends & 8 & $4 \%$ & 2 & $1 \%$ \\
Stakeholder Training & 5 & $2 \%$ & 18 & $7 \%$ \\
Technology & 3 & $1 \%$ & 1 & $<1 \%$ \\
\hline
\end{tabular}

Table 3 identifies the frequencies and percentages of publications across $A P A Q$ and $E C$ over the ten-year span by topical focus. Since $A P A Q$ seeks scholarly publications whose inquiry relates to physical activity and performance for individuals with disability, the journal included a larger percentage of publications pertaining to topics categorized as exercise science $(32 \%)$. Conversely, EC has only $1 \%$ of publication in this topic. The primary topical category of $E C$ is the education/instructional approaches (60\%) category. This percentage was larger than education/instructional approaches publications in $A P A Q(15 \%)$. Discrepancies in exercise sciences and educational/instructional approaches categories were expected prior to this analysis due to the nature and scope of each of the academic journals.

In both journals, topics relating to psychology/sociology were popular $32 \%$ for $A P A Q ; 27 \%$ for $E C)$. Further, both journals included few publications related to research trends/methods (less than 5\%) and technology (1\% or less). Similar to the population of interest categories, $E C$ had more articles pertaining to stakeholder training $(n=18)$ than $A P A Q(n=5)$. This may also be attributed to the more educational focus of the $E C$ journal.

Collectively, trends in population of interest and topical focus may not be mutually exclusive. Rather, social demands often influence both items at the same time. For example, in the United States, recent legislative changes may have influenced both participants and topical areas of publications in $E C$. The reauthorization of the Individuals with Disabilities Education Act addressed the need for highly qualified educators; therefore, more demands may have influenced more publications dealing with teacher and paraprofessional training. In addition, this reauthorization provided new provisions for specific learning disabilities and, in turn, strategies and approaches to identify specific learning disabilities are now being addressed (Bradley, Danielson, \& Doolittle, 2005). APA may be less influenced by changes in education-related legislations than SE, because school-based adapted physical education is only one area within the composition of what is considered APA.

\section{CONCLUSIONS}

The purpose of this documentary analysis was to examine and compare trends in APA and SE publications over a ten-year span. To the knowledge of the authors of this paper, this is the first cross-disciplinary comparative documentary analysis which analyzes publications between APA and SE. Several similarities and differences were found between APA and SE research conducted over the past ten years. The similarities include a heavy reliance on group designs and low numbers of intervention research and explicitly identified theoretical frameworks. Differences were found in several categories including populations of interest and research focus. The findings of this cross-disciplinary document analysis indicate that previous suggestions regarding the use of a theoretical framework (Porretta \& Sherrill, 2005) and the use of non-group designs such as single subject designs (Zhang et al., 2006) in APA research are not reflected in current APA research and may also be applicable to other 
research pertaining to individuals with disabilities in the SE context.

\section{Perspective}

This first-if-its-kind analysis demonstrates characteristics of current APA and SE research which can influence future research. For example, this analysis agrees with Karkaletsi and colleagues (2012) in regard to APA researchers using more qualitative research, a trend that is also evident in SE. However, other suggestions made by APA scholars, such as the further use of single subject designs (e.g., Bouffard, 1993), are not reflected in recent publications. The more prevalent use of these designs (48\% of intervention studies) in $\mathrm{SE}$ research demonstrates that single subject designs can be successful when researching populations that may have low numbers or heterogeneous groups, two challenges that are ubiquitous to SE and APA.

Also, while a recent analysis of publications found research in $A P A Q$ to be highly theoretical (Karkaletsi et al., 2012), this analysis found conflicting evidence which aligns with other previous publications and indicates a dearth of theoretical frameworks (e.g., Haegele \& Porretta, 2015). To enhance future research, those individuals in master's level or doctoral training programs should discuss theoretical/conceptual frameworks with students and suggest courses which introduce single subject designs to their graduate level students.

\section{REFERENCES}

Bradley, R., Danielson, L., \& Doolittle, J. (2005). Response to intervention. Journal of Learning Disabilities, 38, 485-486.

Byiers, B. J., Reichle, J., \& Symons, F. J. (2012). Single-subject experimental design for evidence-based practice. American Journal of Speech-Language Pathology, 21(4), 397-414.

Bouffard, M. (1993). The perils of averaging data in adapted physical activity research. Adapted Physical Activity Quarterly, 10(4), 371-391.
Cervantes, C. M., \& Taylor, W. C. (2011). Physical activity interventions in adult populations with disabilities: A review. Quest, 63(4), 685-410.

Cooper, J. O., Heron, T. E., \& Heward, W. L. (2007) Applied behavior analysis. ( $2^{\text {nd }}$ ed.) Upper Saddle River, NJ: Pearson Prentice Hall.

DePauw, K. (1986). Research on sport for athletes with disabilities. Adapted Physical Activity Quarterly, 3, 292-299.

Depauw, K.P. \& Sherrill, C. (1994). Adapted physical activity: Present and future. Physical Education Review, 17(1), 6-13.

Duncan, D.F. (1989). Content analysis in health education research: An introduction to purposes and methods. Health Education, 20(2), 27-31.

Fraenkel, J., Wallen, N., \& Hyun, H.H. (2012). How to design and evaluate research in education. (8th ed.). Boston: McGraw Hill.

Gay, L. R., Mills, G. E., \& Airasian, P. (2006). Educational research competencies for analysis and applications. ( $8^{\text {th }}$ ed.). Upper Saddle River, NJ: Pearson.

.Haegele, J. A., Lee, J., \& Porretta, D. L. (2015). Research trends in Adapted Physical Activity Quarterly from 2004 to 2013. Adapted Physical Activity Quarterly, 32(3), 187-205.

Haegele, J. A. \& Porretta, D. L. (2015). Physical activity and school-aged individuals with visual impairments: A literature review. Adapted Physical Activity Quarterly, 32(1), 68-82,

Hodge, S. R., Kozub, F. M., Robinson, L. E., \& Hersman, B. L. (2007). Reporting gender, race, ethnicity, and sociometric status: Guidelines for research and professional practice. Adapted Physical Activity Quarterly, 24(1), 21-37.

Horner, R. H., Carr, E. G., Halle, J., McGee, G., Odom, S., \& Wolery, M. (2005). The use of single-subject research to identify evidence-based practice in special education. Exceptional Children, 71(2), 165-179.

Hutzler, Y. (2006). Evidence based research in adapted physical activity: Theoretical and data-based considerations. Revista $d a$ Sobama, 11(1), 13-24. 
Hutzler, T. (2011). Evidence-based practice and research: A challenge to the development of adapted physical activity. Adapted Physical Activity Quarterly, 28(3), 189-209.

Johnson, E.B. (2014). Autism spectrum disorders: The worldwide charm and challenge of autism spectrum disorders. In Anthony F. Rotatori, Jeffrey P. Bakken, Sandra Burkhardt, Festus E. Obiakor, Umesh Sharma (Eds.). Special Education International Perspectives: Biopsychosociol, Cultural, and Disability Aspects (pp.117-151). Bradford, UK: EmeraldGroup Publishing.

Johnson, P. H., \& Kittleson, M. J. (2000). A content analysis of health education teaching strategy/idea articles. Journal of Health Education, 31(5), 292-298.

Johnston, J. M., \& Pennypacker, H. S. (2009). Strategies and tactics of behavior research. ( $3^{\text {rd }}$ ed.). New York, NY: Routledge.

Karkaletsi, F., Skordilis, E.K., Evaggelinou, C., Grammatopoulou, E., \& Spanaki, E. (2012). Research trends in adapted physical activity on the base of APAO journal (2006-2010). European Journal of Adapted Physical Activity, 5(2), 45-64.

Kudlacek, M. \& Barrett, U. (2011). Adapted physical activity as a profession in Europe. European Journal of Adapted Physical Activity, 4(2), 7-16.

Lavay, B., \& Lasko-McCarthey, P. (1992). Adapted physical activity research issues and recommendations. Adapted Physical Activity Quarterly, 9(3), 189-196.

Lee, J. \& Porretta, D.L. (2013). Document analysis of sports literature for individuals with disabilities. Perceptual \& Motor Skills: Physical Development \& Measurement, 116(3), 847-858.

Mastroperi, M. \& Scruggs, T.E. (2014). Report to the readership: Exceptional Children, Volume 80. Exceptional Children, 81(1), 7-10.

McCubbin, J. (2014). Adapted physical activity: Influential impacts to establish a field of study. Kinesiology Review, 3(1), 53-58.

Obrusnikova, I., \& Rocco Dillon, S. (2011). Challenging situations when teaching children with autism spectrum disorder in general physical education. Adapted Physical Activity Quarterly, 28(2), 113131.

O'Connor, J., French, R., Sherrill, C., \& Babcock, G. (2001). Scholarly productivity in adapted physical activity pedagogy: A bibliometric analysis. Adapted Physical Activity Quarterly, 18(4), 434-450.

Porretta, D. L. \& Sherrill, C. (2005). APAQ at twenty: A documentary analysis. Adapted Physical Activity Quarterly, 22(2), 119135.

Reid, G. (2003). Defining Adapted Physical Activity, in Steadward, Wheeler \& Watkinson (Eds.), Adapted Physical Activity, Alberta: University of Alberta Press.

Reid, G., \& Broadhead, G. (1995). APAQ at ten: A documentary analysis. Adapted Physical Activity Quarterly, 12(2), 199126.

Reid, G. \& Prupas, A. (1998). A documentary analysis of research priorities in disability sport. Adapted Physical Activity Quarterly, 15(2), 168-178.

Reid, G. \& Stanish, H. (2003). Professional and disciplinary status of adapted physical activity. Adapted Physical Activity Quarterly, 20(3), 213-229.

Sherrill, C. (2004). Adapted physical activity, recreation and sport: Cross disciplinary and lifespan. ( $6^{\text {th }}$ ed.). Dubuque, IA: Brown \& Benchman.

Sherrill, C. \& Hutzler, Y. (2008). Adapted physical activity sciences. In J. Borms (Ed.), Directory of sport science $\left(5^{\text {th }}\right.$ ed.)(pp.89-103). Berlin, Germany: ICSSPE/ CIEPSS

Thomas, J. R., Nelson, J. K., \& Silverman, S. J. (2005). Research methods in physical activity. (5th ed.). Champaign, IL: Human Kinetics.

Ward, P., \& Ko, B. (2006). Publication trends in the journal of teaching in physical education 1981-2003. Journal of Teaching in Physical Education. 25(3), 266-280.

Watkinson, E. J. \& Wasson, D. L. (1984). The use of single-subject time series designs in adapted physical activity. Adapted Physical Activity Quarterly, 1(1), 19-29. 
Zhang, J., deLisle, L., \& Chen, S. (2006). Analysis of AAHPERD research abstracts published under special populations from
1968 to 2004. Adapted Physical Activity

Quarterly, 23(2), 203-217. 